\title{
A 69-year-old woman with a linear rash
}

A 69-year-old Chinese woman presented with pruritic lesions in the form of linear keratotic papules on the left inframammary region for the past 2 years. On examination, there were multiple oval to round, brown, firm, keratotic papules with distinct scaly edges separated from the surrounding healthy skin (Fig. 1). She was treated with mometasone furoate ointment every morning and topical calcipotriol ointment every night for about 7 months with mild improvement.

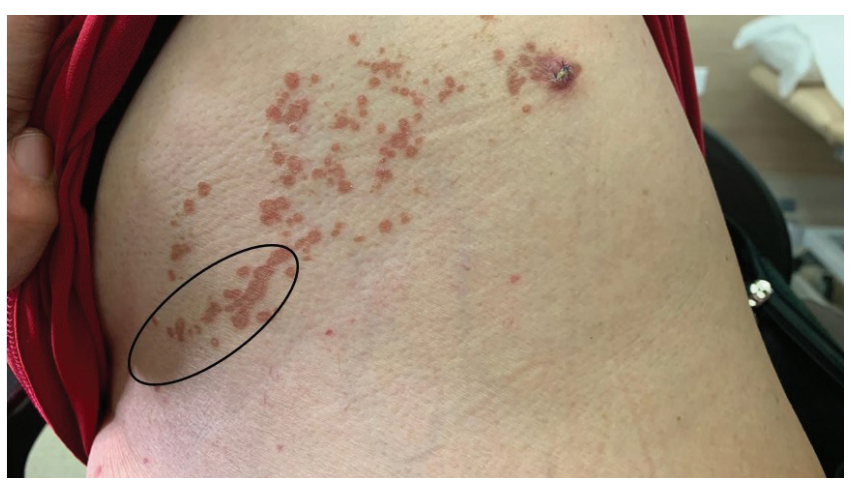

Fig. 1. Multiple brown keratotic papules at the left inframammary region. (Colour figure available online.)

What is the diagnosis?
A. Confluent and reticulated papillomatosis
B. Darier's disease
C. Epidermal naevus
D. Linear porokeratosis
E. Viral warts

A punch biopsy was performed because of the persistent rash despite treatment. Histological examination revealed the presence of cornoid lamella featuring angulated parakeratosis, focal loss of granular layer and vacuolated keratinocytes at the base (Fig. 2). This finding is consistent with the diagnosis for porokeratosis.

Porokeratosis is an uncommon skin disorder of epidermal keratinisation. It is characterised by one or more annular hyperkeratotic plaques with atrophic centres and elevated, thread-like ridges that expand centrifugally. The formation of the cornoid lamella observed histopathologically corresponds to the clinical manifestation of the elevated hyperkeratotic border in porokeratosis. The cornoid lamella is a thin column of tightly packed parakeratotic cells within a keratin-filled epidermal invagination. The apex of the column angles away from the centre of the lesion, and the base of the column demonstrates dyskeratotic keratinocytes and interruption of the epidermal granular layer. ${ }^{1}$

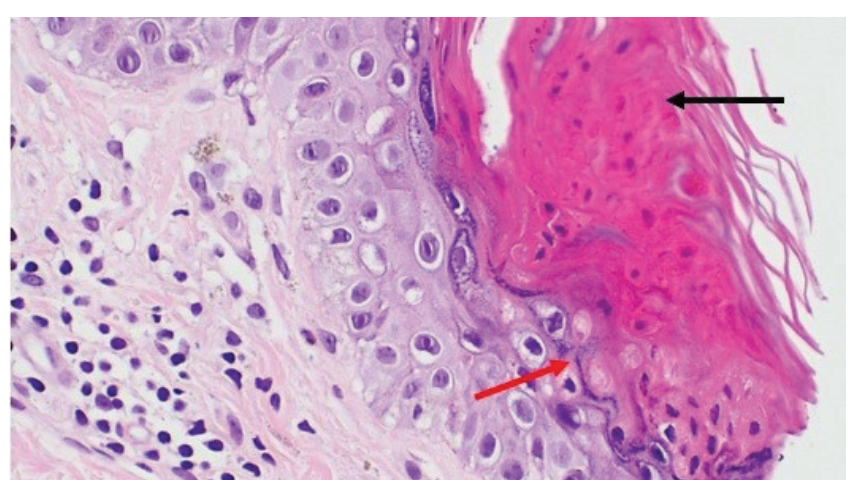

Fig. 2. Haematoxylin and eosin (x400 magnification power) showing porokeratosis. The top arrow indicates the angulated parakeratotic tier, denoting cornoid lamella. The bottom arrow indicates the base of the cornoid lamella, which is vacuolated with focal loss of granular layer. (Colour figure available online.)

Epidermal naevus results from proliferation of epidermal keratinocytes. It is distributed along the lines of Blaschko, where cells migrate as the skin develops before birth. It manifests at birth or in early childhood, which makes it unlikely in our patient.

Another differential diagnosis is viral warts, which are benign growths caused by the human papillomavirus. They can appear as hyperkeratotic papules that are distributed on various surfaces of the body. It is less likely for numerous viral warts to be distributed in a dermatomal pattern. Porokeratotic lesions exhibit a characteristic ridge on its border and a central furrow. On the other hand, pinpoint dots can be seen in viral warts, which represent thrombosed microvessels.

Confluent and reticulated papillomatosis is an uncommon skin condition that typically occurs in young adults. It is characterised by multiple $1-5 \mathrm{~mm}$, hyperpigmented, scaly macules or papillomatous papules in a net-like configuration, affecting the trunk, neck and axillae. Patients are usually asymptomatic. Dermatomal presentation is uncommon.

Darier's disease is an autosomal dominant condition with multiple discrete scaly or greasy papules. Affected sites include seborrheic areas of the face such as scalp margins, forehead, ears, around the nostrils, sides of 
nose, eyebrows and beard area. It can also affect the central chest, neck, back and skinfolds such as the axilla, groin and inframammary region. Symptoms usually appear in late childhood or early adulthood.

Porokeratosis can be classified into localised and generalised forms. The localised forms include porokeratosis of Mibelli, linear porokeratosis and punctate porokeratosis. The generalised variants include disseminated superficial porokeratosis, disseminated superficial actinic porokeratosis and disseminated palmoplantar porokeratosis. ${ }^{2}$

Linear porokeratosis is a rare, unilateral variant with grouped lesions varying in size and number, may involve the entire hemibody, and slowly grow to form irregular annular plaques with well-demarcated raised borders. The central portion may have anhidrosis and alopecia.

Linear porokeratosis appear to have an increased risk of transformation into squamous cell carcinoma or basal cell carcinoma, both of which may be exacerbated by immunosuppression, ultraviolet (UV) light, or irradiation. Fatal outcomes from disseminated squamous cell carcinoma associated with porokeratosis have been reported. Protein p53 expression has been considered as a possible marker of malignant degeneration in patients with porokeratosis. ${ }^{3}$ Malignant transformation of porokeratosis is uncommon. A Singapore study by Tan et al. found that $3.2 \%$ of patients with porokeratosis subsequently developed Bowen's disease and squamous cell carcinoma over various sites of porokeratosis. The duration prior to detection of malignancy ranged from 10 months to 13 years, with a mean of 3.6 years. ${ }^{2}$ Patients with porokeratosis should be given advice on sun protection through the use of broad-spectrum sunscreen and clothing with UV protection. Follow-up for monitoring of lesions is recommended due to the risk of malignancy. Signs to look out for include increased size of lesions, changes in colour, and thickened papules or plaques that can spontaneously ulcerate or bleed.

Treatment options for porokeratosis include topical corticosteroids, oral and topical retinoids, topical vitamin D3 analogues, keratolytic agents, 5-fluorouracil, topical imiquimod 5\% cream and topical diclofenac gel. Surgical options include excision, electrodesiccation and curettage, cryotherapy, dermabrasion and laser therapy. ${ }^{1}$

Our patient was treated with $0.025 \%$ tretinoin cream. However, she developed an irritant contact dermatitis. Hence, tretinoin was discontinued after 1 month's use. The patient was switched to Daivobet ointment

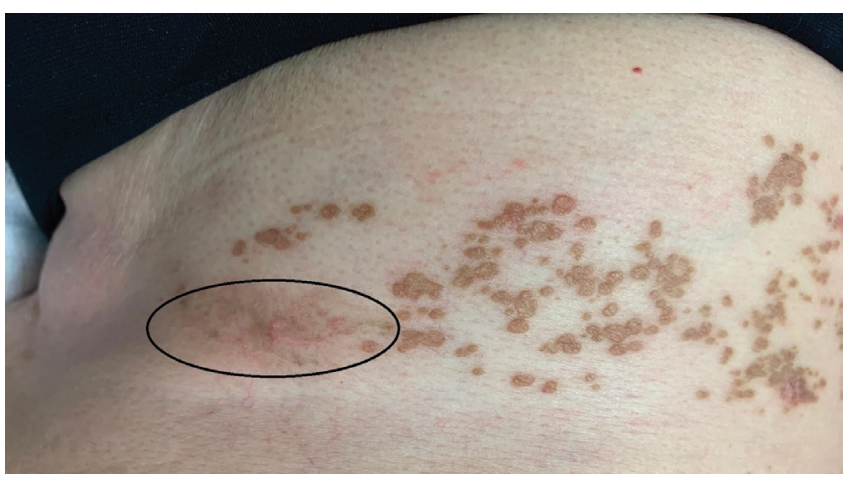

Fig. 3. Post-inflammatory hyperpigmentation (circled) after lesions were treated with liquid nitrogen therapy. (Colour figure available online.)

(betamethasone dipropionate and calcipotriol) twice daily leading to flattening of the lesions. Daivobet ointment was used as the patient had significant pruritus and skin inflammation as a result of the prior use of $0.025 \%$ tretinoin cream. Long-term use of potent topical corticosteroids can lead to local adverse effects such as striae, cutaneous atrophy and telangiectasia. Our patient used the Daivobet ointment for 2 weeks and tolerated treatment well, without side effects. Her treatment was subsequently changed to liquid nitrogen therapy after the irritant contact dermatitis resolved. She had 2 sessions of liquid nitrogen therapy, performed once every 2 months. As shown in Fig. 3, this has resulted in resolution of the lesions with postinflammatory hyperpigmentation.

\section{Acknowledgement}

Dr Sze-Hwa Tan from the Department of Laboratory Medicine, Changi General Hospital assisted in obtaining histology pictures for this case report.

\section{REFERENCES}

1. Weidner $\mathrm{T}$, Illing $\mathrm{T}$, Miguel $\mathrm{D}$, et al. Treatment of Porokeratosis: A Systematic Review. Am J Clin Dermatol 2017;18:435-49.

2. Tan LS, Chong WS. Porokeratosis in Singapore: an Asian perspective. Australas J Dermatol 2012;53:e40-4.

3. Escanilla-Figueroa C, Jimeno-Ortega I, Fuenzalida-Wong H, et al. Generalized linear porokeratosis. An Bras Dermatol 2018;93:477-8.

Siqing Ee ${ }^{1}{ }_{M R C P(U K)}$, Yong-Kwang Tay ${ }^{1}{ }_{F R C P(L o n d)}$

${ }^{1}$ Department of Dermatology, Changi General Hospital, Singapore

Correspondence: Dr Siqing Ee, Department of Dermatology, Changi General Hospital, 2 Simei Street 3, Singapore 529989.

Email: siqing.ee@mohh.com.sg 